\title{
Combating resistance while maintaining innovation: the future of antimicrobial stewardship
}

\author{
Richard J Vickers ${ }^{*}$ (1D), Matteo Bassetti ${ }^{2}$, Cornelius J Clancy ${ }^{3}$, Kevin W Garey ${ }^{4}$, David E \\ Greenberg $^{5}$, Minh-Hong Nguyen ${ }^{3}$, David Roblin ${ }^{1}$, Glenn S Tillotson ${ }^{6}$ \& Mark H Wilcox ${ }^{7}$ \\ 'summit Therapeutics, Abingdon, UK \\ ${ }^{2}$ Infectious Diseases Clinic, Department of Health Sciences, University of Genoa, Genoa \& Hospital Policlinico San Martino, Genoa, \\ Italy \\ ${ }^{3}$ University of Pittsburgh, Division of Infectious Diseases, Pittsburgh, PA, USA \\ ${ }^{4}$ Department of Pharmacy Practice \& Translational Research, University of Houston College of Pharmacy, Houston, TX, USA \\ ${ }^{5}$ Department of Internal Medicine, University of Texas Southwestern Medical Center, Dallas, TX, USA \\ ${ }^{6}$ GST Micro, Richmond, VA, USA \\ ${ }^{7}$ Department of Microbiology, Leeds Teaching Hospitals \& University of Leeds, Leeds, UK \\ *Author for correspondence: richard.vickers@summitplc.com
}

Antimicrobial resistance represents a significant global health threat. However, a commercial model that does not offer a return on investment resulting in a lack of investment in antibiotic R\&D, means that the current pipeline of antibiotics lacks sufficient innovation to meet this challenge. Those responsible for defining, promoting and monitoring the rationale use of antibiotics (the antimicrobial stewardship programme) are key to addressing current shortcomings. In this personal perspective, we discuss the future role stewardship can play in stimulating innovation, a need to move away from a pharmacy budget dominated view of antibiotic use, and the impact of the ever-increasing sophistication and interdisciplinary nature of antimicrobial control programs. Changes are needed to optimize clinical outcomes for patients.

First draft submitted: 31 July 2019; Accepted for publication: 30 August 2019; Published online:

17 September 2019

Keywords: antibiotic cost • antimicrobial stewardship • novel mechanism antibiotics

Antibiotics represent one of the most important components of the modern medical armamentarium. However, the inevitable development of antimicrobial resistance has resulted in an alarming prevalence of multidrug-resistant organisms. Despite the clear medical need for new agents, the clinical development pipeline for antibiotics is sparse. Except for bedaquiline for MDR-TB, no antibiotics with a novel mechanism of action have been approved for human use since daptomycin in 2002. While valuable agents have been approved since, which address specific resistance mechanisms or provide alternative pharmacokinetics such as dalbavancin and the other lipoglycopeptides, these drugs are analogs of known classes that offer incremental benefit over other class members (Table 1). They are typically broad-spectrum agents, developed for more than one indication and often for labeled indications with low unmet medical need; characteristics that may promote resistance. Continued development of next generation drugs is only a stop-gap approach, since resistance mechanisms to these classes already exist and bacteria are only one short step away from adapting to the latest analog [1]. For example, resistance to ceftazidime/avibactam and ceftolozane/tazobactam emerged soon after approval, and indeed before frequent prescribing of these agents had occurred $[2,3]$. In addition, newer generations of antibiotics have not always produced beneficial drugs, as shown by the relative toxicity of many later fluoroquinolones, resulting in market withdrawals.

A key contributor to the lack of novel antibiotics is that commercial returns have been meager, and the sector is increasingly considered as unattractive to developers, resulting in a lack of investment and innovation. New antibiotics are perceived as 'break-glass-in-emergency' medicines that we should all have but use only in the direst of emergencies. To help address this, several 'push' mechanisms have been established to support R\&D [4]. While significantly contributing to much-needed investment, they do not address the commercial issues and various 


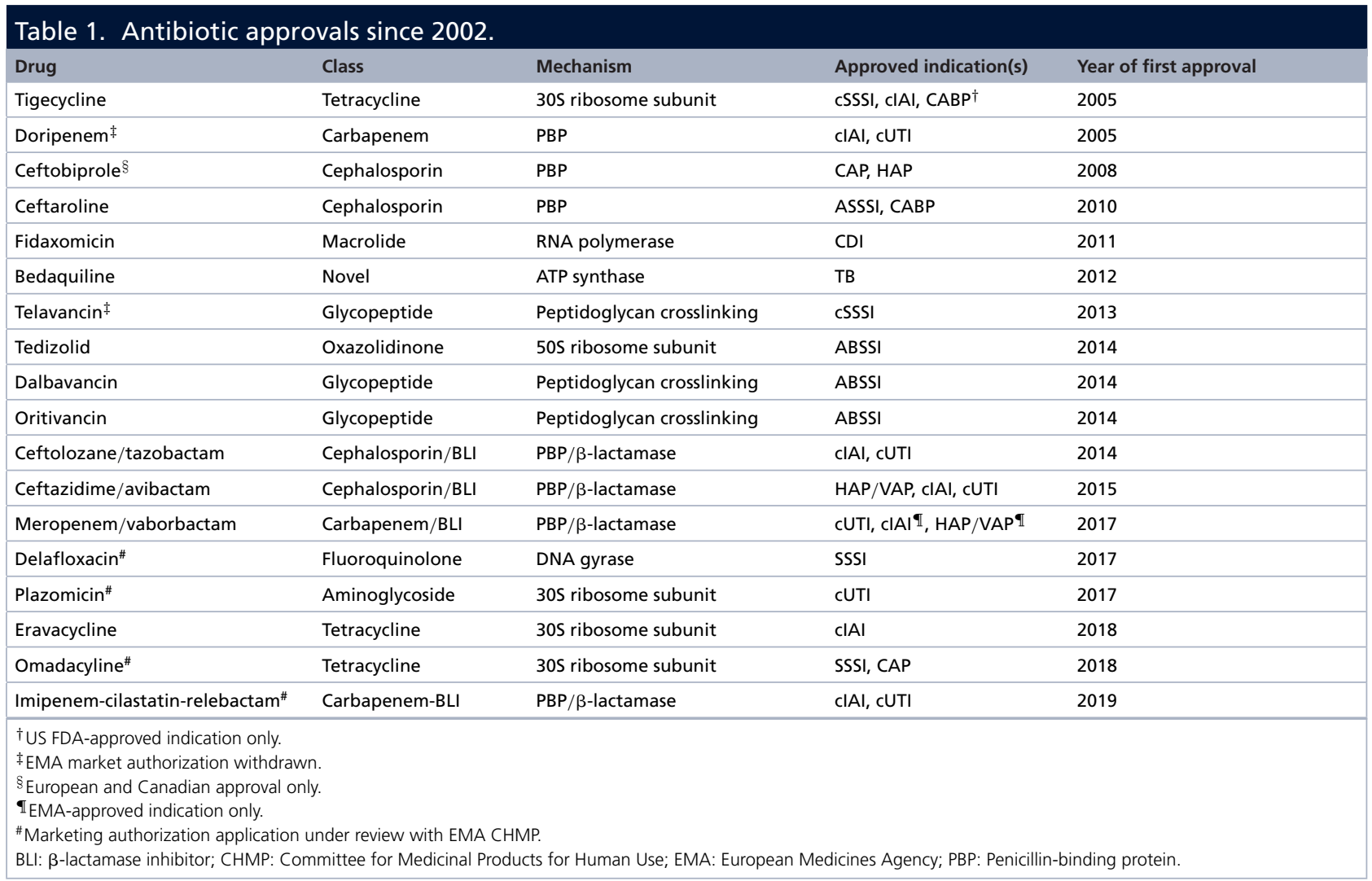

economic models ('pull' mechanisms) have been suggested, with most based on a Market Entry Reward (MER) - a form of economic prize on approval of a new antibiotic [5]. While efforts made to propose such models are welcome, implementation in the near future is uncertain. The MERs require significant political and societal will and, importantly, international collaboration. How they could be implemented (e.g., at a global or national level) and who would control these is unknown. Other solutions have been proposed such as creating nonprofit organizations to take over the role of discovery and development of antibiotics [6]. However, as for MERs the funding and sustainability of such organizations, along with building the required skills and resources, poses significant challenges.

\section{The role of antimicrobial stewardship}

Given both the emergence of resistance and lack of new agents, there has been increased focus on the prudent use of antibiotics. From the 1970s, when programs to control antibiotic use emerged through the initial proposal of the term 'stewardship' [7], antimicrobial stewardship programs (ASP) have become central to treating infections. The National Action Plan issued by the White House [8], called for ASPs to be established in all acute care hospitals by 2020 and by 2016, 64.2\% of US hospitals had met the CDC core elements of stewardship [9]. In Europe, numerous initiatives have been instigated at European wide, national and regional levels [10], with ESGAP, the ESCMID Study Group for antimicrobial stewardship, playing a particularly active role.

The term stewardship has evolved over time [11], and today numerous definitions of stewardship exist (Box 1). All describe a multidisciplinary approach (including pharmacists, physicians, microbiologists and infection control) to the appropriate use of antibiotics to optimize patient outcomes by ensuring the right drug, dose and duration are administered. As recommended by The Infectious Diseases Society of America (IDSA) [12], core to ASPs is either a preauthorization and/or prospective audit and feedback (PAF) system. Restrictive preauthorization programs require clinicians to secure approval from an ASP to use certain antibiotics while PAF proactively engages with the physician once the antibiotic has been prescribed. Effective examples of preauthorization programs have been reported [13,14]. However, they require real time authorization, which is often not available, and restriction alone may cause issues unless implemented with a high degree of skill as reviewed by Garau [15]. In particular, delays 
Box 1. Definitions of antimicrobial stewardship.

- Infectious Diseases Society of America, SHEA, PIDS Consensus statement: "coordinated interventions designed to improve and measure the appropriate use of (antibiotic) agents by promoting the selection of the optimal (antibiotic) drug regimen including dosing, duration of therapy and route of administration."

- CDC: "antimicrobial stewardship programs can optimize treatment of infections and antibiotic use - with the goal to provide every patient with the right antibiotics, at the right time, at the right dose and for the right duration - to reduce adverse events associated with antibiotics and improve patient outcomes."

- NICE: "an organizational or healthcare-system-wide approach to promoting and monitoring judicious use of antimicrobials to preserve their future effectiveness."

- WHO: "framework to preserve antimicrobial medicines by taking measures to promote their control, appropriate distribution as well as appropriate use."

- Duke Center for Antimicrobial Stewardship and Infection Prevention: our mission remains driven by two goals to improve patient safety and enhance quality of care.

to appropriate therapy and placing undue access barriers to physician's optimal choice of therapy may lead to poorer clinical outcomes, including excess mortality. Examples of the impact of inappropriate therapy include a recent systematic review and meta-analysis of outcomes in severe Gram-negative infections showing an increased risk of mortality (adjusted OR: 3.30; 95\% CI: 2.42-4.49) [16], and a retrospective cohort study of the premier perspective database (2009-2013) of carbapenem resistance and Enterobacteriaceae infections also showing increased mortality (12.2 vs $9.9 \%$; $\mathrm{p}<0.001)$ [17]. Restriction of an agent may result in the 'squeeze the balloon effect', first coined by Burke [18]. For example, Rahal observed that a preauthorization requirement for cephalosporins reduced the incidence of ceftazidime-resistant Klebsiella spp. by 44\%, but was associated with a concomitant increase in imipenem use and a 68.7\% increase in the incidence of imipenem-resistant Pseudomonas aeruginosa [19]. Thus, restriction can lead to both desirable and undesirable consequences, and Rahal later emphasized the need to 'squeeze the balloon' at multiple sites [20].

PAF approaches proactively engage with physicians to educate and inform while maintaining prescribing freedom. Direct comparisons between the approaches are few, although a recent Cochrane review [21] noted preauthorization approaches could be detrimental to communication between clinical and ASP teams and ultimately concluded enablement approaches (e.g. PFA) consistently increased the effect of ASP interventions. This has been noted by others [22]. A Cochrane meta-analysis also noted restrictive interventions were more effective only when the need is urgent [23]. Tamma et al. showed, in a quasi-experimental crossover trial comparing restriction or PFA, that PFA was more effective at reducing antibiotic usage, determined by days of therapy (DOTs), with median values of eight and six DOT per 1000 patient-days $(\mathrm{p}=0.03)$ for restrictive and PFA approaches, respectively [24].

To assess an ASP's impact, performance measures are required covering clinical, microbiological, consumption and financial elements $[25,26]$. The IDSA guidelines recommend the best measure is monitoring antibiotic use, ideally by DOTs, with expenditure assessed on prescriptions. However, the latter only measures drug acquisition costs, and not total care costs that represent a more meaningful economic indicator [12]. To truly meet the objectives of ASPs, which should be to ensure that judicious antimicrobial use optimizes clinical outcomes, measures such as resistance, mortality, readmissions, length of stay and oral switch would be more meaningful. However, the complexity of collecting and interpreting such data often restricts ASPs to focusing on antibiotic usage and acquisition cost, despite a measured reduction in antibiotic usage not confirming a successfully implemented ASP; it is simply a process outcome $[27,28]$. Spellberg and colleagues discussed how to justify implementation of impactful ASPs in a way that resonates with healthcare administrators, who are invariably dealing with budgets requiring implementation of one program to be at the expense of spend in other areas [29]. To those within, and expert on, ASPs metrics such as antibiotic usage may enable them to understand the wider benefits of stewardship and convey success messages, but it creates a perception stewardship is primarily about reducing costs. That perception is held, not only by those outside of the healthcare system, but potentially by some physicians whose prescribing decisions may be unduly influenced toward economic, and away from clinical outcome, considerations. A recent survey of 1044 IDSA EIN members indicated that $87 \%$ of hospital providers considered cost reduction a major driver of ASPs [30]. This finding is stark, given antibiotic costs are not mentioned in ASP definitions. Similarly, a survey of ASPs in 82 acute care institutions in Florida reported the most common outcome measure by 56 facilities was drug purchasing costs (70\%); only 43, 38 and 34\% of facilities assessed patient outcomes, LOS, and adverse drug reactions, respectively. In addition, of perceived reasons for antimicrobial misuse, $84 \%$ of clinicians feared being 
sued for untreated infections [31]; further evidence of the undue pressures on prescribers promoting inappropriate antibiotic use. A recent worldwide review of studies, including many from European countries, that assessed the impact of quality measures in ASPs noted only 13 of 63 studies reported on patient outcomes such as mortality and length of stay. In contrast, 21 of the 63 studies reported only on cost savings and antimicrobial usage with the remainder reporting on resistance and/or CDI rates [32]. In 2019, The Consensus on Antimicrobial Stewardship Evaluations study group published a comprehensive systematic review of the quality of antimicrobial stewardship studies as part of a process to develop recommendations to improve the quality of such studies [33]. The findings clearly showed that the design quality of stewardship evaluations was poor, had not improved during the period under review (1950-2017) and that the majority reported on process outcomes only. For hospital studies, 46 and $72 \%$ did not report on clinical or microbiological outcomes, respectively; in other words, the outcome measures that would most meaningfully describe an ASPs benefit to patients. While well-developed and sophisticated ASPs have reported high quality studies and assessments [34], the results reported by the Consensus on Antimicrobial Stewardship Evaluation study group suggest a different picture in the majority of ASPs.

As noted by many, measuring ASP performance purely on acquisition costs results in policies naturally restricting more expensive, yet potentially superior agents driving overutilization of less effective drugs [35,36]. As discussed by Cunha, restriction should only be for agents with high resistance potential [37], and low cost antibiotics may have higher overall total costs when considering Clostidium difficile infection (CDI), resistance and poorer clinical outcomes. A review by Emberger $e t$ al. described two important expert stewardship panels that were convened to define outcome metrics and that neither recommended cost [28].

Acquisition cost metrics are likely used as they are probably the simplest to gather yet if financial considerations are to be made they should be focused on reducing unneeded antibiotic use, on total care costs and not solely on acquisition costs; especially as healthcare reforms are shifting toward quality of care and away from fee-for-service models [38]. The CDC's most recent report on antibiotic use in the US highlights, despite improvements, continued high level use of unnecessary antibiotics with approximately 47 million antibiotic courses prescribed each year for infections that do not require antibiotic therapy and that often the recommended agents and duration of therapy were not selected [39]. This continued inappropriate antibiotic use not only contravenes the principles of stewardship putting patients at risk of harm but is a significant financial and resource drain.

Perhaps this describes why that, despite stewardship aiming to ensure antibiotics are used to optimize clinical outcomes, there are areas where this appears not to be happening. Carbapenem-resistant Enterobacteriaceae (CREs) are among the most worrisome drug resistant pathogens [40], and effective agents against these infections have recently been approved and others are in late stage development [41]. A Phase III study comparing meropenem/vaborbactam with best available therapy in patients with suspected or confirmed CRE infection at a range of body sites (cUTI/AP, HABP, VABP, cIAI and bacteraemia) was halted on the recommendation of the Data and Safety Monitoring Board (DSMB) due to higher cure rates, and reduced mortality and nephrotoxicity in meropenem/vaborbactam recipients [42]. Although the sample size was small, the data were encouraging; estimated improvement in cure at TOC was 32.7\% (95\% CI: 4.6-60.8) associated with an estimated $17.7 \%$ (95\% CI: $-44.7-9.3)$ reduction in day 28 mortality. Similar superiority for cure and mortality have been reported with other agents including ceftazidime/avibactam [43,44]. Kadri et al. recently proposed a phenotypic susceptibility definition of difficult-totreat resistance (DTR) defined as resistance to at least one member of all $\beta$-lactam classes and fluoroquinolones; in other words, agents considered first-line, high efficacy and low toxicity. Mortality following BSI caused by DTR (typically treated with tigecycline, aminoglycosides or colistin) compared with non-DTR Gram-negative bacteria was $40 \%$ higher; adjusted mortality risk $1.4(95 \%$ CI: $1.2-1.6$; $p<0.001)$ [45]. These data are important evidence of the adverse patient impact of using less efficacious and potentially more toxic antibiotics. However, uptake of these new agents into practice is unacceptably slow. A recent study across 132 US hospitals, using a clinical administration database, showed that the median time to first use (range) of ceftazidime/avibactam and ceftolozane/tazobactam was 463 days ( 48 to $>1200$ ) and 405 days $(13$ to $>1268$ ), respectively [46]. In another study, investigators using IQVIA antibiotic prescription data estimated that more CRE infections in the USA were treated with an intravenous polymyxin than a new anti-CRE agent (ceftazidime/avibactam, meropenem/vaborbactam and plazomicin) from February 2018 to January 2019 (Figure 1) [47]. Use of new agents did not exceed that of intravenous polymyxins until December 2018. These data were discordant with results from a survey of US hospital-based pharmacists, which indicated that the new agents were positioned as first-line against CRE urinary tract and nonurinary tract infections at approximately 50 and $90 \%$ of centers, respectively. Anti-CRE drugs were estimated to treat only 


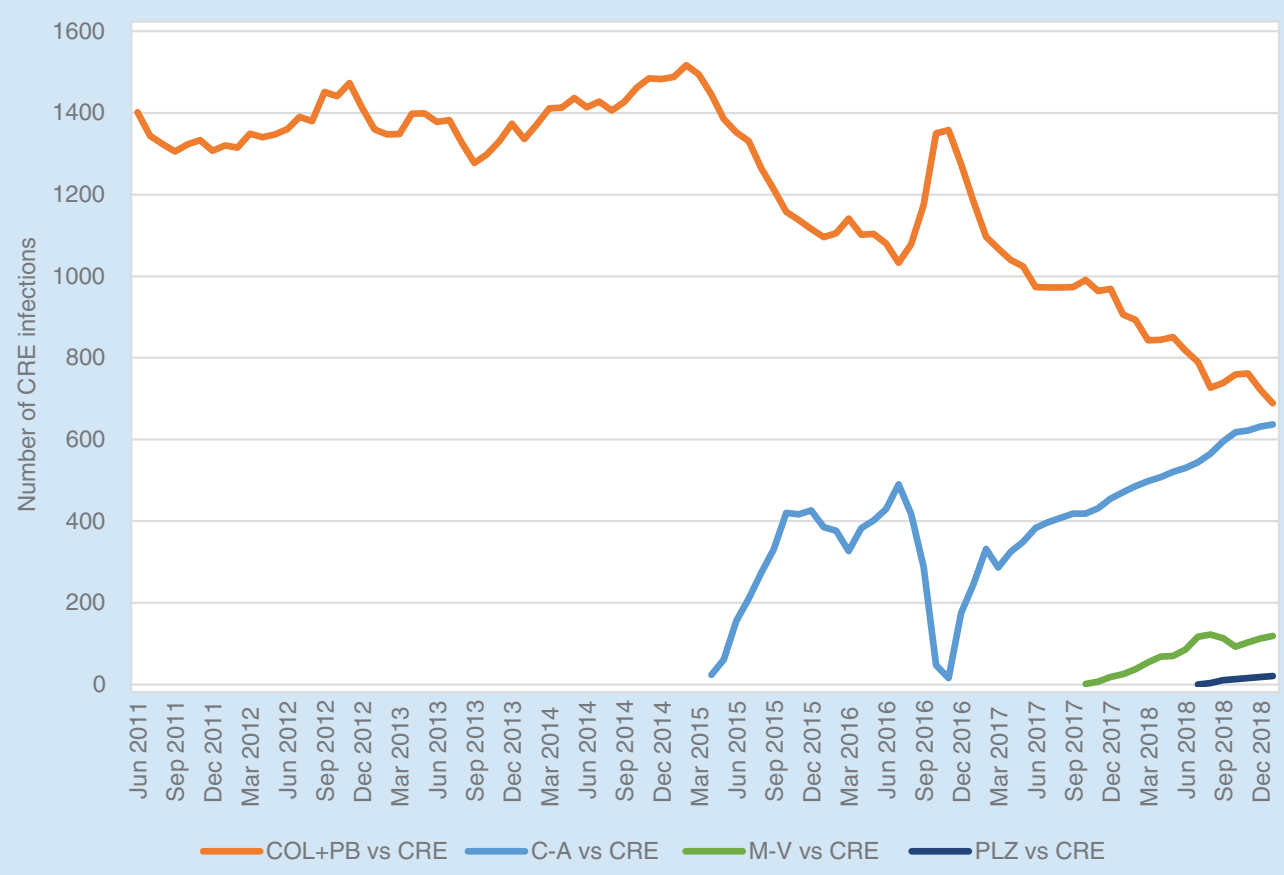

Figure 1. Estimated number of colistin, polymyxin B, ceftazidime/avibactam, meropenem/vaborbactam and plazomicin treatment courses for carbapenem-resistant Enterobacteriaceae infections.

C-A: Ceftazidime-avibactam; COL: Colistin; CRE: Carbapenem-resistant Enterobacteriaceae; M-V: Meropenem-vaborbactam; PB: Polymyxin B; PLZ: Plazomicin.

35\% (range: 23-62\%) of CRE infections in which they were expected to be first-line agents. Clearly, research on behavioral and economic factors that impact use of the new drugs and polymyxins is needed.

If continued reliance on colistin is due to restriction of more efficacious, less toxic, but more expensive agents, then we are failing in our responsibilities to severely ill, vulnerable patients. Colistin, a drug made available prior to the 1962 US FDA Kefauver-Harris Drug Amendments requiring proof of safety and efficacy for FDA marketing approval, would be unlikely to receive regulatory approval today, is not primum non nocere, and use should be restricted beyond that of the newly available therapies

$\mathrm{CDI}$ is another scenario characterized by frequent prescribing of older antibiotics despite alternatives with better patient outcomes being available. CDIs are often treated with oral vancomycin or metronidazole and in the case, of metronidazole, we know that this option is inferior to vancomycin [48]. Oral vancomycin raises potential resistance issues with VRE selection and our awareness of the gut microbiota as a reservoir of resistance mechanisms and source of infection continues to increase [49]. In addition, vancomycin is associated with unacceptable rates of recurrent $\mathrm{CDI}$, induced by collateral damage to the gut microbiota during therapy [50]. If preventing resistance and reducing CDI rates are targets for ASPs, then restricting oral vancomycin would seem sensible. However, we see restrictions to the use of fidaxomicin, a drug shown to reduce rates of recurrent CDI when used frontline [51,52], to potentially reduce 28 day all-cause mortality [53], and is not associated with colonization or spread of VRE [54]. The often-cited 'justification' for this scenario is the high acquisition cost of fidaxomicin despite considerable evidence demonstrating wider health economic benefits [55-58].

\section{Innovation \& stewardship}

This all leads to a paradox. We want to prescribe the patient the right drug at the right time to provide the best clinical outcome while minimizing unwanted downstream consequences; in other words, adhering to the principles of stewardship. However, the absence of novel mechanism agents, and the fear of resistance emerging to those few new agents we do have, often means stewardship considerations result in restricted access to appropriate medicines. This was highlighted in Miller's commentary on the approval of delafloxacin, describing a hypocritical 
stance of begging for, but then shunning use of, new antibiotics [59]. Whether perception or reality, if ASPs are primarily about reducing drug acquisition costs, the situation is exacerbated by deterring investment resulting in a dwindling pipeline. Pull incentives are a possible solution but challenges to their implementation, longevity and assumption of continued significant formulary restriction continue to deter investment. Economic considerations, while important, are not the principle purpose of ASPs and drug acquisition costs do not represent true wider pharmacoeconomic benefit.

The situation is further compounded when evidence-based decisions to use an antibiotic are reliant on noninferiority (NI) trials. The NI test, which aims to establish that a new experimental treatment is not unacceptably less efficacious than an active control treatment already in use [60,61], is currently required in settings beyond the most serious and drug-resistant of infections as it is unethical to randomize patients to either placebo or an inferior comparator. A new antibacterial may successfully meet the prespecified NI test and achieve regulatory approval but the data pose challenges - the new drug appears to be 'just as good' or 'no worse' than the comparator which may unfortunately limit its use [62,63]. This can be a somewhat superficial view. In certain settings, it may be impossible to run anything other than a NI test on the primary end point, and so a more holistic view of the data by ASPs and other groups is required to understand the true value of a new agent and its benefits for patients. This could include data beyond initial cure such as wider pharmacoeconomic benefits, reduced readmissions and reduced infection recurrence. However, in settings of particularly high unmet medical need superiority studies should be possible as discussed by Powers et al. [64].

So how to resolve this conundrum and ensure that we, rightly, meet the overarching principles of stewardship? Key is innovation - a shift away from the traditional approach of broad-spectrum agents, often from existing classes and developed for multiple indications. Novel mechanism agents developed for specific infections to address clear unmet needs aligns with stewardship goals. Such agents would have a spectrum of activity targeted toward the pathogens of concern, be developed through clinical trials that show clear benefits over standard of care and developed for use only in a specific infection or indication and not as 'jack-of-all-trades' agents. These would be the appropriate therapies targeted to a specific patient need that stewardship requires, limiting the requirement for excessive formulary restriction.

Multiple examples of where this precision approach could be adopted exist. For example, infections due to Neisseria gonorrhoea are almost universally treated with ceftriaxone (often in combination with azithromycin), an agent that should be used for severe infections, such as meningitis, cIAI and HAP [65]. Given global prevalence of 106 million cases annually, and the ability of $N$. gonorrhoeae to develop, harbor and spread resistance, the utility of ceftriaxone for indications where its use is more appropriate is threatened [66]. A novel class antibiotic for gonorrhoea, developed and used only to treat gonorrhoea, would address a significant public health concern and preserve ceftriaxone for use in severe infections.

Such an approach does come with significant scientific challenges. Identification of new classes of antibiotics has proven difficult yet there is clear progress in this area. As of October 2017, 13 of the 17 projects funded by CARB-X were novel mechanisms, chemotypes or approaches to treat bacterial infections [67], and more have been funded subsequently. The Pew Trust identified 42 antibiotics in clinical development of which ten could be considered novel [68], including murepavadin, a novel anti-Pseudomonas specific drug, now entering Phase III trials [69]. In addition, numerous innovative and nontraditional technologies are emerging such as antisense, engineered bacteriophages and microbiota-based therapies [70].

We can also now capitalize on regulatory innovation in trial designs. As part of the 21 st Centuries Cures Act, the Limited Population Antimicrobial Drug pathway emerged allowing for smaller, more feasible trials in limited populations [71]. The recent positive opinion adopted by the Committee for Medicinal Products for Human Use on meropenem/vaborbactam for the treatment of cUTI, cIAI and HAP/VAP on the basis of a large Phase III study in cUTI [72], and a study in 77 subjects with confirmed or suspected CRE infection at a range of body sites [42], further demonstrates the welcome and forward-thinking approach by regulators to help address the challenge of antimicrobial resistance (AMR). Many other active and expert groups continue to propose innovative approaches to clinical trials $[73,74]$.

The use of such targeted antibiotics could be enabled on several fronts, led by increasingly sophisticated ASPs. For example, the University Medical Centre in Groningen uses a model that integrates antimicrobial stewardship, diagnostic stewardship and infection control into a patient centric, personalized care plan aimed at optimizing clinical outcomes [75]. Pharmacists should have a more strategic role beyond contracting and containing the cost of antimicrobials. Pharmacists with infectious diseases knowledge can impact on inappropriate antimicrobial 
regimens through various strategies, including optimizing prescribing behavior, monitoring antimicrobial use, infection prevention and education, training and public engagement [76]. Stewardship not only requires use of the appropriate drug, but also timely and appropriate dosing, sometimes aided by therapeutic drug monitoring (TDM) which is increasingly recognized as important in treating infections in the critically ill [77-79]. To be effective, TDM requires an individualized patient approach using a complex dataset; precision antibiotics for personalized treatment could benefit from TDM, but factors such as access to drug assays and poor integration of PK software with medical records, represent common barriers to effective implementation [80].

Major technological advances are potentially precipitating a revolution in rapid diagnostics providing pathogen identification and susceptibility in hours not days. This has been extensively reviewed by others describing benefits of their use in improving outcomes, including impacts on mortality [81-84]. Importantly, the benefits of rapid diagnostics requires integration into ASPs to ensure correct use of tests, interpretation of results and again requires close discussion between the microbiology lab, physician and wider ASP team to ensure timely action [82,84]. Intriguingly, an oft-cited barrier to implementation of new diagnostic technologies is cost - prescribing a broadspectrum generic antibiotic is simply cheaper than using a rapid diagnostic. This is another key example of the challenges balancing immediate budget impact with wider cost and patient benefits.

Finally, if trial designs capture important outcomes data, such as reductions in unplanned interventions and total care costs, it is then incumbent on payors and providers, including ASPs, to consider such data from both individual patients and a population health perspective. We acknowledge, however, that more guidance is needed to optimize such approaches.

\section{Future perspective}

Antimicrobial resistance continues to advance and has reached unprecedented levels presenting a significant challenge to modern medicine and patient welfare. However, a commercial model that does not offer a return on the significant expenditure required to develop new, and especially truly innovative, antibiotics is stifling progress. What could the future hold if we are to address the antimicrobial resistance crisis? A new way of developing antibacterials, focused on novel mechanisms addressing specific unmet medical needs and developed to address specific indications; a clear shift away from additional generations of established antibiotic classes that are often broad-spectrum agents developed for multiple indications. A vibrant pipeline of such precision agents could alleviate resistance concerns and provide clinicians with the targeted therapies they require to deliver the best outcomes for patients. Used in more upfront settings, or at least not the very last resort, the commercial returns would improve, promoting further R\&D investment and limit the need for excessive restriction. However, this requires urgent action by all parties involved in tackling infectious diseases, including drug developers, healthcare providers and policy makers. Although efforts to address the commercial issues at the policy level have been slow, and the reality of large MERs uncertain, we are highly encouraged to see the UK take a lead, though the NHS and NICE, with a pilot scheme assessing a value-based model for antibiotic reimbursement [85]. However, the qualification criteria for an antibiotic to be adopted as part of such a scheme, and the quantum of commercial return, remain unclear. In addition, the UK alone cannot address these issues and a consistent global approach is required. Through all of this, stewardship should remain a central component of our approach to infection management, but its core aim of optimizing outcomes for patients should be re-emphasized.

\section{Author contributions}

All authors conceived the manuscript. RJ Vickers drafted the first version of the manuscript and conducted initial literature searches. All authors contributed equally to review and drafting of subsequent version.

\section{Financial \& competing interests disclosure}

RJ Vickers is an employee and holds share options in Summit Therapeutics. M Bassetti has received personal fees from Angelini, AstraZeneca, Bayer, Biomerieux, Cidara, Cubist, Pfizer, Melinta Therapeutics, Menarini, MSD, Nabriva, Paratek, Roche, Shionogi, Tetraphase, The Medicines Company and Astellas Pharma Inc. CJ Clancy has been awarded investigator initiated research grants from Astellas, Merck, Melinta, and Cidara for projects unrelated to this study, served on advisory boards or consulted for Astellas, Merck, the Medicines Company, Cidara, Scynexis, Shionogi, Qpex and Needham \& Company, and spoken at symposia sponsored by Merck and T2Biosystems. KW Garey has received personal fees from Merck, Paratek, Biomerieux and Seres and received grants from Summit Therapeutics, Merck, Paratek and Tetraphase. DE Greenberg has received grants from Shionogi. MH Nguyen has been awarded investigator initiated research grants from Astellas, Merck, Melinta and Cidara for projects unrelated to this study, 
and served on advisory boards for Astellas, Merck, the Medicines Company, Scynexis and Shionogi. D Roblin is an employee and holds share options in Summit Therapeutics. GT has received personal fees from Melinta, Shionogi, KBP Biosciences, Spero, Sinsa Pharmaceuticals and TechLabs. MH Wilcox has received personal feSEes from Abbott Laboratories, Actelion, Antabio, AiCuris, Alere, Astellas, AstraZeneca, Bayer, Biomèrieux, Cambimune, Cerexa, Cubist, Da Volterra, The European Tissue Symposium, Ferring, The Medicines Company, Menarini, Merck, Meridian, Motif Biosciences, Nabriva, Paratek, Pfizer, Phico Therapeutics, Qiagen, Roche, Seres, Spero, Surface Skins, Sanofi-Pasteur, Summit, Synthetic Biologics and Valneva and has received grants from Abbott, Actelion, Astellas, Biomèrieux, Cubist, Da Volterra, Merck, MicroPharm, Morphochem AG, Motif Biosciences, Nabriva, Paratek, Pfizer, SanofiPasteur, Seres, Summit and The European Tissue. The authors have no other relevant affiliations or financial involvement with any organization or entity with a financial interest in or financial conflict with the subject matter or materials discussed in the manuscript apart from those disclosed.

No writing assistance was utilized in the production of this manuscript.

\section{Open access}

This work is licensed under the Attribution-NonCommercial-NoDerivatives 4.0 Unported License. To view a copy of this license, visit http://creativecommons.org/licenses/by-nc-nd/4.0/

\section{Executive summary}

\section{The problem of resistance coupled with an absence of innovation}

- The widespread emergence of high-level antimicrobial resistance represents a major medical crisis.

- The pipeline of new antibiotics, especially innovative agents with new mechanisms of action addressing the most worrisome of resistant pathogens, is sparse at best.

Aims, perceptions \& realities of antimicrobial stewardship programs

- To address the issue of resistance, the concept of antimicrobial stewardship was developed to ensure the prudent use of antibiotics to minimize resistance development and ensure the clinical longevity of the agents we have.

- The overarching aim of antimicrobial stewardship programs (ASPs) is a multidisciplinary approach to optimizing clinical outcomes for patients through the use of the right antibiotic, in the right patient and at the right time.

- Despite many highly sophisticated ASPs being established that successfully result in improved clinical metrics such as reduced resistance, reduced $C$. difficile infections and better patient outcomes, all too often ASPs are measuring success on process metrics and minimizing drug acquisition costs.

- A broken commercial model for antibiotics and a pharmacy budget driven approach to stewardship, whether perceived or actual, is stifling investment and innovation in novel antibiotic R\&D resulting in the real threat of the future returning to the pre-antibiotic era.

Personal perspective on one aspect of how to address the antimicrobial resistance crisis

- In this personal perspective, we propose that development of new mechanism, targeted and precision antibacterials developed to address major unmet medical needs, is required to address the AMR crisis and that an innovative approach to antimicrobial stewardship must play a fundamental role in helping to stimulate innovation.

- It is incumbent on all those involved with infectious diseases, from drug developers to pharmacists, physicians and administrators, to provide patients with life-threatening infections the medicines they need.

\section{References}

Papers of special note have been highlighted as: $\bullet$ of interest; $\bullet \bullet$ of considerable interest

1. Coates AR, Halls G, Hu Y. Novel classes of antibiotics or more of the same? Br. J. Pharmacol. 163(1), 184-194 (2011).

2. Shields RK, Nguyen MH, Press EG, Chen L, Kreiswirth BN, Clancy CJ. Emergence of ceftazidime-avibactam resistance and restoration of carbapenem susceptibility in Klebsiella pneumoniae Carbapenemase-producing K pneumoniae: a case report and review of literature. Open Forum Infect. Dis. (2017). https://www.ncbi.nlm.nih.gov/pmc/articles/PMC5493938/

3. MacVane SH, Pandey R, Steed LL, Kreiswirth BN, Chen L. Emergence of ceftolozane-tazobactam-resistant Pseudomonas aeruginosa during treatment is mediated by a single AmpC structural mutation. Antimicrob. Agents Chemother. 61(12), e01183-01117 (2017).

4. Simpkin VL, Renwick MJ, Kelly R, Mossialos E. Incentivising innovation in antibiotic drug discovery and development: progress, challenges and next steps. J. Antibiot. 70(12), 1087-1096 (2017).

5. Ardal C, Rottingen JA, Opalska A, Van Hengel AJ, Larsen J. Pull incentives for antibacterial drug development: an analysis by the transatlantic task force on antimicrobial resistance. Clin. Infect. Dis. 65(8), 1378-1382 (2017).

6. Nielsen TB, Brass EP, Gilbert DN, Bartlett JG, Spellberg B. sustainable discovery and development of antibiotics - is a nonprofit approach the future? N. Engl. J. Med. 381(6), 503-505 (2019). 
7. McGowan J, Gerding D. Does antibiotic restriction prevent resistance. New Horizons 4(3), 370-376 (1996).

8. CDC. White House National action plan for combating antibotic resistant bacteria. (2015) https://www.cdc.gov/drugresistance/pdf/national_action_plan_for_combating_antibotic-resistant_bacteria.pdf

9. CDC. Core elements of hospital antibiotic stewardship programs. (2014) http://www.cdc.gov/getsmart/healthcare/implementation/core-elements.html

10. Pulcini C. Antibiotic stewardship: a European perspective. FEMS Microbiol. Lett. 364(23), 1-4 (2017).

11. Dyar OJ, Huttner B, Schouten J, Pulcini C. Esgap. What is antimicrobial stewardship? Clin. Microbiol. Infect. 23(11), 793-798 (2017).

12. Barlam TF, Cosgrove SE, Abbo LM et al. Implementing an antibiotic stewardship program: guidelines by the Infectious Diseases Society of America and the Society for Healthcare Epidemiology of America. Clin. Infect. Dis. 62(10), e51-77 (2016).

13. White AC Jr, Atmar RL, Wilson J, Cate TR, Stager CE, Greenberg SB. Effects of requiring prior authorization for selected antimicrobials: expenditures, susceptibilities, and clinical outcomes. Clin. Infect. Dis. 25(2), 230-239 (1997).

14. Pakyz AL, Oinonen M, Polk RE. Relationship of carbapenem restriction in 22 university teaching hospitals to carbapenem use and carbapenem-resistant Pseudomonas aeruginosa. Antimicrob. Agents Chemother. 53(5), 1983-1986 (2009).

15. Garau J. Impact of antibiotic restrictions: the ethical perspective. Clin. Microbiol. Infect. 12(Suppl. 5), 16-24 (2006).

16. Raman G, Avendano E, Berger S, Menon V. Appropriate initial antibiotic therapy in hospitalized patients with gram-negative infections: systematic review and meta-analysis. BMC Infect. Dis. 15, 395-406 (2015).

17. Zilberberg MD, Nathanson BH, Sulham K, Fan W, Shorr AF. Carbapenem resistance, inappropriate empiric treatment and outcomes among patients hospitalized with Enterobacteriaceae urinary tract infection, pneumonia and sepsis. BMC Infect. Dis. 17(1), 279-292 (2017).

18. Burke JP. Antibiotic resistance-squeezing the balloon? JAMA 280(14), 1270-1271 (1998).

19. Rahal JJ, Urban C, Horn D et al. Class restriction of cephalosporin use to control total cephalosporin resistance in nosocomial Klebsiella. JAMA 280(14), 1233-1237 (1998).

20. Rahal JJ, Urban C, Segal-Maurer S. Nosocomial antibiotic resistance in multiple gram-negative species: experience at one hospital with squeezing the resistance balloon at multiple sites. Clin. Infect. Dis. 34(4), 499-503 (2002).

21. Davey P, Marwick CA, Scott CL et al. Interventions to improve antibiotic prescribing practices for hospital inpatients. Cochrane Database Syst. Rev. 2, CD003543 (2017).

22. Reed EE, Stevenson KB, West JE, Bauer KA, Goff DA. Impact of formulary restriction with prior authorization by an antimicrobial stewardship program. Virulence 4(2), 158-162 (2013).

23. Davey P, Brown E, Charani E et al. Interventions to improve antibiotic prescribing practices for hospital inpatients. Cochrane Database Syst. Rev. (4), CD003543 (2013).

24. Tamma PD, Avdic E, Keenan JF et al. What is the more effective antibiotic stewardship intervention: preprescription authorization or postprescription review with feedback? Clin. Infect. Dis. 64(5), 537-543 (2017).

25. Dik JW, Hendrix R, Poelman R et al. Measuring the impact of antimicrobial stewardship programs. Ex. Rev. Anti-infect. Ther. 14(6), 569-575 (2016).

26. Brotherton AL. Metrics of antimicrobial stewardship programs. Med. Clin. North Am. 102(5), 965-976 (2018).

27. Dodds Ashley ES, Kaye KS, DePestel DD, Hermsen ED. Antimicrobial stewardship: philosophy versus practice. Clin. Infect. Dis. 59(Suppl. 3), S112-S121 (2014).

28. Emberger J, Tassone D, Stevens MP, Markley JD. The current state of antimicrobial stewardship: challenges, successes, and future directions. Curr. Infect. Dis. Rep. 20(9), 31 (2018).

29. Spellberg B, Bartlett JG, Gilbert DN. How to pitch an antibiotic stewardship program to the hospital C-suite. Open Forum Infect. Dis. 3(4), (2016).ofw210

30. Johannsson B, Beekmann SE, Srinivasan A, Hersh AL, Laxminarayan R, Polgreen PM. Improving antimicrobial stewardship: the evolution of programmatic strategies and barriers. Infect. Control Hosp. Epidemiol. 32(4), 367-374 (2011).

31. Abbo L, Lo K, Sinkowitz-Cochran R et al. Antimicrobial stewardship programs in Florida's acute care facilities. Infect. Control Hosp. Epidemiol. 34(6), 634-637 (2013).

32. Akpan MR, Ahmad R, Shebl NA, Ashiru-Oredope D. A review of quality measures for assessing the impact of antimicrobial stewardship programs in hospitals. Antibiotics 5(1), 5-21 (2016).

33. Schweitzer VA, van Heijl I, van Werkhoven $\mathrm{CH}$ et al. The quality of studies evaluating antimicrobial stewardship interventions: a systematic review. Clin Microbiol Infect 25(5), 555-561 (2018).

- Discussion on the often poor quality of studies assessing effectivness of antimicrobial stewardship programs.

34. Baur D, Gladstone BP, Burkert F et al. Effect of antibiotic stewardship on the incidence of infection and colonisation with antibiotic-resistant bacteria and Clostridium difficile infection: a systematic review and meta-analysis. Lancet Infect. Dis. 17(9), 990-1001 (2017). 
35. McKellar MR, Fendrick AM. Innovation of novel antibiotics: an economic perspective. Clin. Infect. Dis. 59(Suppl. 3), S104-S107 (2014).

36. Parente DM, Morton J. Role of the pharmacist in antimicrobial stewardship. Med. Clin. North Am. 102(5), 929-936 (2018).

37. Cunha CB, Varughese CA, Mylonakis E. Antimicrobial stewardship programs (ASPs): the devil is in the details. Virulence 4(2), 147-149 (2013).

38. Nagel JL, Stevenson JG, Eiland EH 3rd, Kaye KS. Demonstrating the value of antimicrobial stewardship programs to hospital administrators. Clin. Infect. Dis. 59(Suppl. 3), S146-S153 (2014).

39. US Department of Health and Human Services C. Antibiotic use in the united states, 2018 update: progress and opportunities. Atlanta, GA (2019).

40. Bassetti M, Poulakou G, Ruppe E, Bouza E, Van Hal SJ, Brink A. Antimicrobial resistance in the next 30 years, humankind, bugs and drugs: a visionary approach. Intensive Care Med. 43(10), 1464-1475 (2017).

41. Petty LA, Henig O, Patel TS, Pogue JM, Kaye KS. Overview of meropenem-vaborbactam and newer antimicrobial agents for the treatment of carbapenem-resistant Enterobacteriaceae. Infect. drug Dis. 11, 1461-1472 (2018).

42. Wunderink RG, Giamarellos-Bourboulis EJ, Rahav G et al. Effect and safety of meropenem-vaborbactam versus best-available therapy in patients with carbapenem-resistant enterobacteriaceae infections: the TANGO II randomized clinical trial. Infect. Dis. Ther. (2018).

- A randomized clinical trial showing clear superiority of meropenem/vaborbactam over alternative therapies in the treatment of infections due to carbapenem-resistant Enterobacteriaceae.

43. Shields R. Ceftazidime-avibactam is superior to other treatment regimens against carbapenem-resistant Klebsiella pneumoniae bacteremia. Antimicrob. Agents Chemother. 61(8), e00883-00817 (2017).

44. van Duin D, Lok JJ, Earley M et al. Colistin versus ceftazidime-avibactam in the treatment of infections due to carbapenem-resistant Enterobacteriaceae. Clin. Infect. Dis. 66(2), 163-171 (2018).

45. Kadri SS, Adjemian J, Lai YL et al. Difficult-to-treat resistance in gram-negative bacteremia at 173 US hospitals: retrospective cohort analysis of prevalence, predictors, and outcome of resistance to all first-line agents. Clin. Infect. Dis. 67(12), 1803-1814 (2018).

46. Schulz LT, Kim SY, Hartsell A, Rose WE. Antimicrobial stewardship during a time of rapid antimicrobial development: potential impact on industry for future investment. Diagn. Microbiol. Infect. Dis. (2019).

47. Clancy CJ, Potoski BA, Buehrle D, Nguyen MH. Estimating the treatment of carbapenem resistant Enterobacteriaceae infections in the United States using antibiotic prescription data. Open Forum Inf. Dis. (2019).

- Clear evidence of extensive colistin use despite availability of superior agents.

48. Johnson S, Louie TJ, Gerding DN et al. Vancomycin, metronidazole, or tolevamer for Clostridium difficile infection: results from two multinational, randomized, controlled trials. Clin. Infect. Dis. 59(3), 345-354 (2014).

49. van Schaik W. The human gut resistome. Philos. Trans. R Soc. Lond. B Biol. Sci. 370(1670), 20140087 (2015).

50. Kelly CP. Can we identify patients at high risk of recurrent Clostridium diffcile infection? Clin. Microbiol. Infect. 18(Suppl. 6), 21-27 (2012).

51. Cornely OA, Crook DW, Esposito R et al. Fidaxomicin versus vancomycin for infection with Clostridium difficile in Europe, Canada, and the USA: a double-blind, non-inferiority, randomised controlled trial. Lancet Infect. Dis. 12(4), 281-289 (2012).

52. Louie TJ, Miller MA, Mullane KM et al. Fidaxomicin versus vancomycin for Clostridium diffcile infection. N. Engl. J. Med. 364(5), 422-431 (2011).

53. Goldenberg SD, Brown S, Edwards L et al. The impact of the introduction of fidaxomicin on the management of Clostridium difficile infection in seven NHS secondary care hospitals in England: a series of local service evaluations. Eur. J. Clin. Microbiol. Infect. Dis. 35(2), 251-259 (2016).

54. Nerandzic MM, Mullane K, Miller MA, Babakhani F, Donskey CJ. Reduced acquisition and overgrowth of vancomycin-resistant enterococci and Candida species in patients treated with fidaxomicin versus vancomycin for Clostridium difficile infection. Clin. Infect. Dis. 55(Suppl. 2), S121-S126 (2012).

55. Rubio-Terres C, Cobo Reinoso J, Grau Cerrato $\mathrm{S}$ et al. Economic assessment of fidaxomicin for the treatment of Clostridium difficile infection (CDI) in special populations (patients with cancer, concomitant antibiotic treatment or renal impairment) in Spain. Eur. J. Clin. Microbiol. Infect. Dis. 34(11), 2213-2223 (2015).

56. Gallagher JC, Reilly JP, Navalkele B, Downham G, Haynes K, Trivedi M. Clinical and economic benefits of fidaxomicin compared to vancomycin for Clostridium diffcile Infection. Antimicrob. Agents Chemother. 59(11), 7007-7010 (2015).

57. Nathwani D, Cornely OA, Van Engen AK, Odufowora-Sita O, Retsa P, Odeyemi IA. Cost-effectiveness analysis of fidaxomicin versus vancomycin in Clostridium difficile infection. J. Antimicrob. Chemother. 69(11), 2901-2912 (2014).

58. Reveles KR, Backo JL, Corvino FA, Zivkovic M, Broderick KC. Fidaxomicin versus vancomycin as a first-line treatment for clostridium difficile-associated diarrhea in specific patient populations: a pharmacoeconomic evaluation. Pharmacotherapy 37(12), 1489-1497 (2017). 
59. Miller LG. Another new antibiotic for skin infections and why infectious disease specialists are hypocrites. Clin. Infect. Dis. 68(7), 1223-1224 (2018).

60. Powers JH, Fleming TR. Noninferiority trials: clinical understandings and misunderstandings. Clin. Investig. (Lond.) 3(3), 215-218 (2013).

61. DiNubile MJ. Noninferior antibiotics: when is "not bad" "good enough"? Open Forum Infect. Dis. 3(3), (2016).ofw110

62. Herath C, Blondeau JM. Do we really understand what we want or need out of antimicrobial stewardship programs. Future Med. Clin. Pract. 10(1), 5-9 (2013).

63. Deak D, Outterson K, Powers JH, Kesselheim AS. Progress in the fight against multidrug-resistant bacteria? A review of U.S. Food and Drug Administration-approved antibiotics, 2010-2015. Ann. Intern. Med. 165(5), 363-372 (2016).

64. Powers JH, Evans SR, Kesselheim AS. Studying new antibiotics for multidrug resistant infections: are today's patients paying for unproved future benefits? BMJ 360, k587 (2018).

65. WHO. Model list of essential medicines (2017). https://www.who.int/medicines/publications/essentialmedicines/en/

66. Unemo M, Shafer WM. Antimicrobial resistance in Neisseria gonorrhoeae in the 21 st Century: past, evolution, and future. Clin. Microbiol. Rev. 27(3), 587-613 (2014).

67. Mullard A. Preclinical antibiotic pipeline gets a pick-me-up. Nat. Rev. Drug Dis. 16(11), 741-742 (2017).

68. Pew Trust. Antibiotics currently in global clinical development (2018). https: //www.pewtrusts.org/en/research-and-analysis/data-visualizations/2014/antibiotics-currently-in-clinical-development

69. Martin-Loeches I, Dale GE, Torres A. Murepavadin: a new antibiotic class in the pipeline. Exp. Rev. Anti-infect. Ther. 16(4), 259-268 (2018).

70. Tse BN, Adalja AA, Houchens C, Larsen J, Inglesby TV, Hatchett R. Challenges and opportunities of nontraditional approaches to treating bacterial infections. Clin. Infect. Dis. 65(3), 495-500 (2017).

71. Boucher HW, Ambrose PG, Chambers HF et al. White paper: developing antimicrobial drugs for resistant pathogens, narrow-spectrum indications, and unmet needs. J. Infect. Dis. 216(2), 228-236 (2017).

\section{- Interesting discussion on approaches to trial designs.}

72. Kaye KS, Bhowmick T, Metallidis S et al. Effect of meropenem-vaborbactam vs piperacillin-tazobactam on clinical cure or improvement and microbial eradication in complicated urinary tract infection: the TANGO I randomized clinical trial. JAMA 319(8), 788-799 (2018).

73. de Kraker MEA, Sommer H, de Velde F et al. Optimizing the design and analysis of clinical trials for antibacterials against multidrug-resistant organisms: a white paper from COMBACTE's STAT-Net. Clin. Infect. Dis. 67(12), 1922-1931 (2018).

74. McDonnell A, Rex JH, Goossens H, Bonten M, Fowler VG Jr, Dane A. Efficient delivery of investigational antibacterial agents via sustainable clinical trial networks. Clin. Infect. Dis. 63(Suppl. 2), S57-S59 (2016).

75. Dik JH, Poelman R, Friedrich AW, Niesters HGM, Rossen JWA, Sinha B. Integrated stewardship model comprising antimicrobial, infection prevention, and diagnostic stewardship (AID stewardship). J. Clin. Microbiol. 55(11), 3306-3307 (2017).

-• An excellent description of a highly sophisticated antimicrobial stewardship program.

76. Garau J, Bassetti M. Role of pharmacists in antimicrobial stewardship programmes. Int. J. Clin. Pharm. 40(5), 948-952 (2018).

77. Roberts JA, Abdul-Aziz MH, Lipman J et al. Individualised antibiotic dosing for patients who are critically ill: challenges and potential solutions. Lancet Infect. Dis. 14(6), 498-509 (2014).

78. Vincent JL, Bassetti M, Francois B et al. Advances in antibiotic therapy in the critically ill. Critical Care 20(1), 133-146 (2016).

79. Luyt CE, Brechot N, Trouillet JL, Chastre J. Antibiotic stewardship in the intensive care unit. Critical Care 18(5), 480-492 (2014).

80. Rawson TM, O’Hare D, Herrero P et al. Delivering precision antimicrobial therapy through closed-loop control systems. J. Antimicrob. Chemother. 73(4), 835-843 (2018).

81. Tsalik EL, Bonomo RA, Fowler VG Jr. New molecular diagnostic approaches to bacterial infections and antibacterial resistance. Annu. Rev. Med. 69, 379-394 (2018).

82. Timbrook TT, Spivak ES, Hanson KE. Current and future opportunities for rapid diagnostics in antimicrobial stewardship. Med. Clin. North Am. 102(5), 899-911 (2018).

83. Bauer KA, Perez KK, Forrest GN, Goff DA. Review of rapid diagnostic tests used by antimicrobial stewardship programs. Clin. Infect. Dis. 59(Suppl. 3), S134-S145 (2014).

84. Edmiston CE, Garcia R, Barnden M, DeBaun B, Johnson HB. Rapid diagnostics for bloodstream infections: a primer for infection preventionists. Am. J. Infect. Control 46(9), 1060-1068 (2018).

85. Kmietowicz Z. New antibiotics: NHS will test "pay for usefulness" model to stimulate research. BMJ 366, 14610 (2019). 
